\title{
Final Technical Report for DOE Grant DE-FG02-03ER15473 "Molecular Level Design of Heterogeneous Chiral Catalysis"
}

\author{
PIs: Profs. David S. Sholl and Andrew J. Gellman \\ Department of Chemical Engineering \\ Carnegie Mellon University, Pittsburgh, PA 15213 \\ Email: sholl@andrew.cmu.edu, ag4b@andrew.cmu.edu
}

\begin{abstract}
Other CMU Personnel: Rees Rankin, Joanna James, Layton Baker, Ye Huang (grad students)

Collaborators: Prof. Francisco Zaera (University of California, Riverside), Prof. Wilfred Tysoe (University of Wisconsin, Milwaukee)

Budget period: 9/15/03-11/15/06 (Total period for this award: 9/15/03-11/15/06)

Budget amount: $\$ 800,000$

Project start date: $9 / 15 / 03$

Reporting period: 9/15/03-11/15/06
\end{abstract}

\begin{abstract}
The production of enantiomerically pure chiral compounds is of great importance in the pharmaceutical industry. Although processes involving chiral catalysis and separations involving solid surfaces are known, the molecular-scale details of these processes are not well understood. This lack of understanding strongly limits the development of new chiral processes. Our collaborative research effort examines several intertwined aspects of chirality and enantioselectivity at catalytically active metal surfaces. At Carnegie Mellon, our efforts focus on the development of chirally imprinted metal powders as materials for chiral columns and the experimental and theoretical study of small chiral molecules adsorbed on well-characterized metal surfaces, both achiral and chiral. These efforts are being performed in close collaboration with our team members at the University of California Riverside and the University of Wisconsin Milwaukee.
\end{abstract}




\section{TECHNICAL REPORT}

\section{Motivation and Long-term Goals}

The production of enantiomerically pure chiral compounds for use in the pharmaceuticals industry and other biologically related applications is a $>\$ 100$ billion per year commercial enterprise. Furthermore, the ability to produce enantiomerically pure pharmaceuticals allows the treatment of diseases that would not be possible if such pharmaceuticals were administered in racemic form. Catalysis is a viable route to the production of fine chemicals such as pharmaceuticals and can be achieved enantiospecifically if one can design chiral catalysts that are capable of enantioselectivity. For the most part this is done using homogeneous catalysts based on organometallic compounds continuing heavy metals. The development of enantioselective heterogeneous catalysts offers opportunities to capitalize on the inherent advantages of heterogeneous catalysis. Such materials do exist but there is little understanding of the mechanisms by which they catalyze reactions enantioselectively. The primary goal of this program is to understand the origins of enantioselectivity on chiral catalysts and to exploit this knowledge in the development of new enantioselective catalysts.

The hypothesis underpinning our collaborative research effort is that there are three origins of chirality or enantioselectivity at catalytic metal surfaces:

- natural chirality arising from the atomic structure of the metal surface,

- chirality arising from 'isolated' adsorbed chiral template molecules on the surface that have 1:1 interactions with chiral adsorbates,

- and chirality arising from adsorbates that form chiral ensembles with long range order.

Chiral molecules adsorb and react enantioselectively on such surfaces by interacting with the naturally chiral structure of the surface or via 1:1 interactions with the chiral template or by adsorbing at chiral 'pockets' in the chiral ensemble. Implicit in this hypothesis is the idea that there are three components that define enantiospecificity:

- the substrate surface and its structure,

- the adsorbed template molecule,

- and the chiral probe that adsorbs on the surface.

Our collaborative program attempts to understand the relative importance of each of these three components in enantiospecific adsorption and catalytic reactions.

The work at Carnegie Mellon complements ongoing work at the University of California at Riverside and at the University of Wisconsin at Milwaukee on the enantioselectivity of templated chiral surfaces. In addition, the work at Carnegie Mellon work explores the origins of enantioselectivity at naturally chiral surfaces and focuses on their production in macroscopic quantities for separations and catalytic applications. 


\section{Research Progress}

Our experimental and theoretical research program has examined several aspects of creating and controlling chirality on solid metal surfaces.

\subsection{Experimental studies of chirally templated copper surfaces}

The work in Gellman's lab on the enantioselective adsorption of chiral probe molecules on templated chiral surfaces has built upon the early observations of enantioselective adsorption of R- and S-propylene oxide on $\mathrm{Pd}(111)$ and $\mathrm{Pt}(111)$ surfaces templated with $\mathrm{R}$ - and S-2-butanoxide groups. That work was done by Tysoe and by Zaera [1,2]. The work in Gellman's lab has extended that work to $\mathrm{Cu}$ substrates of different structure and to the use of different chiral probes.

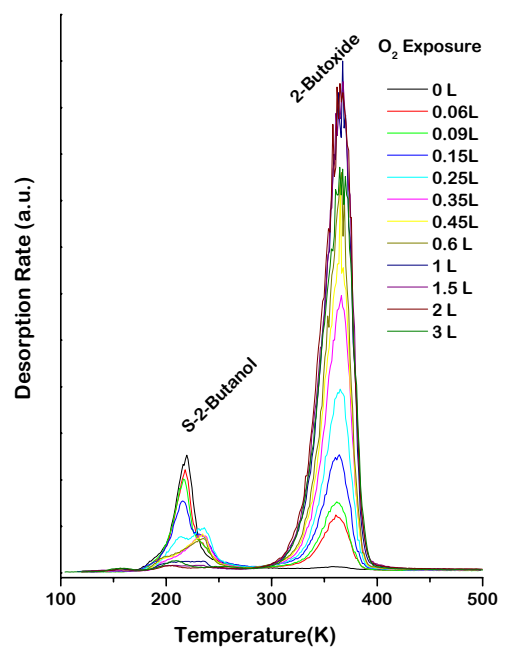

Figure 1. TPD of 2-butanoxide on the $\mathrm{Cu}(100)$ surface at various initial coverages. The peak at 360 $\mathrm{K}$ is the desorption of methylethylketone generated by the decomposition of the 2-butanoxide. The peaks at $\sim 220 \mathrm{~K}$ are the desorption of molecular 2-butanol

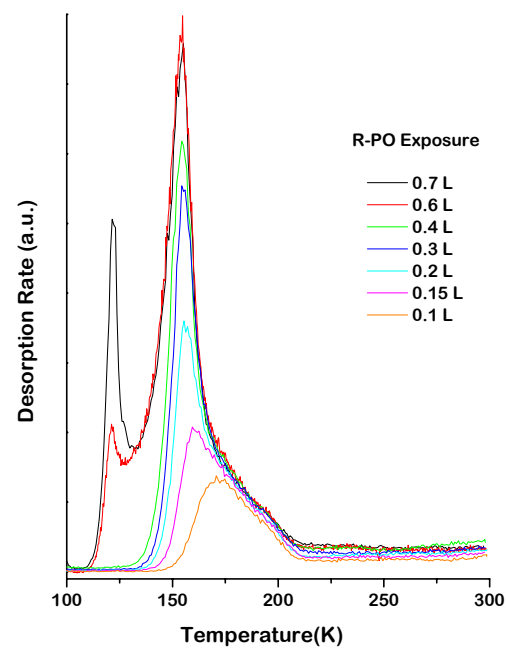

Figure 2. TPD spectra of R-propylene oxide from the clean $\mathrm{Cu}(100)$ surface. Monolayer desorption occurs over the temperature range $150-200 \mathrm{~K}$. Propylene is completely desorbed at temperatures below that at which 2-butanoxide decomposes.

The first system studied was R-propylene oxide on R- and S- 2-butanoxide templated $\mathrm{Cu}(100)$ surface. Figure 2 shows the TPD spectra of 2-butanoxide decomposition during heating on the $\mathrm{Cu}(100)$ surface after adsorption at various different coverages. The coverage of the butanoxy groups is controlled by first adsorbing varying amounts of oxygen on the $\mathrm{Cu}(100)$ surface and then co-adsorbing a monolayer of 2-butanol. The deprotonation of the 2-butanol by the pre-adsorbed oxygen yields that adsorbed 2butanoxy groups on the surface. Figure 3 shows the TPD spectra of propylene oxide from the clean $\mathrm{Cu}(100)$ surface. The important point is that propylene oxide adsorbs and desorbs reversibly and that it is completely desorbed at temperatures well below the onset of 2-butanoxide decomposition. Thus it can be adsorbed and desorbed from the templated surface without the need to regenerate the 2-butanoxide monolayer for each experiment. This make this system significantly simpler to study than propylene oxide 
and 2-butanoxide on $\operatorname{Pt}(111)$ or $\operatorname{Pd}(111)$.

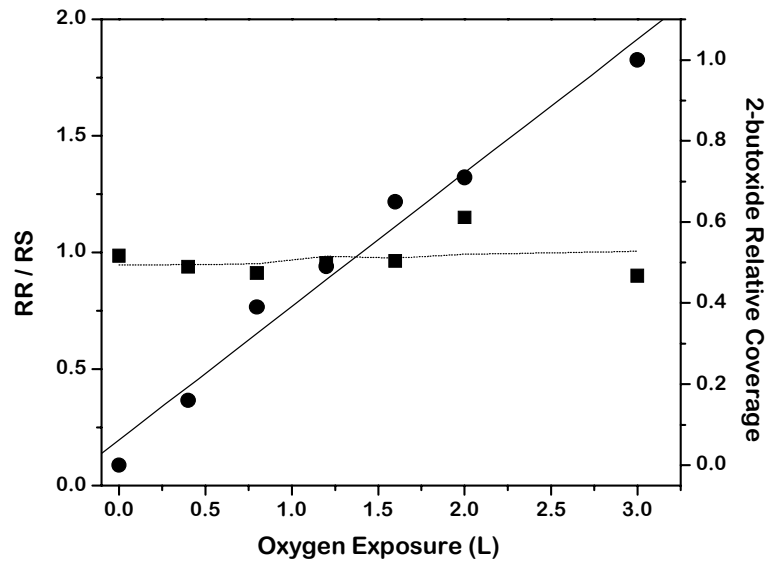

Figure 3. The coverage of 2-butanoxide $(\bullet)$ on the $\mathrm{Cu}(100)$ surface as a function of the oxygen exposure to the surface prior to adsorption of 2-butanol. The ratio of R-propylene oxide adsorbed in the monolayer on the $\mathrm{Cu}(100)$ surface templated with R-2-butanoxy and S-2-butanoxy $(\mathbf{\bullet})$, as a function of the initial oxygen exposure.

Temperature programmed desorption was used to measure the amount of R-and Spropylene oxide that could be adsorbed in the monolayer on $\mathrm{Cu}(100)$ surfaces with templated layers of R-and S-2-butanoxide groups at different coverages. Figure 4 shows the enantiospecific ratio of the amounts of R-propylene oxide adsorbed on both the Rand the S-2-butanoxy templated surfaces. At all 2-butanoxy coverages, the amounts of propylene oxide adsorbed in the monolayers are identical. This is also true on surface templated with R-2-butanoxy. In other words, no enantiospecificity has been detected in the adsorption of R- and S-propylene oxide on $\mathrm{Cu}(100)$ surfaces templated with $\mathrm{R}$ - and $\mathrm{S}$ 2-butanoxide. Table 1 shows the set of adsorbate- substrate systems that have been studied during the course of this work. It is somewhat surprising that we have not been able to detect enantioselective adsorption on chirally templated $\mathrm{Cu}$ surfaces, given the success that has been observed in the work of Tysoe and Zeara's groups on the $\operatorname{Pd}(111)$ and $\operatorname{Pt}(100)$ surfaces $[1,2]$.

Table 1. The set of Cu surfaces, chiral template molecules and chiral probe molecules studied in Gellman's laboratory.

\begin{tabular}{|l|l|l|}
\hline Surface & Template & Probe \\
\hline $\mathrm{Cu}(100)$ & 2-butanoxide & propylene oxide \\
\hline $\mathrm{Cu}(100)$ & 2-butanoxide & R-3-methylcyclohexanone \\
\hline $\mathrm{Cu}(111)$ & 2-butanoxide & propylene oxide \\
\hline $\mathrm{Cu}(111)$ & 2-butanoxide & R-3-methylcyclohexanone \\
\hline $\mathrm{Cu}(111)$ & 2-butanol & propylene oxide \\
\hline $\mathrm{Cu}(111)$ & alaninol & propylene oxide \\
\hline
\end{tabular}




\subsection{Experimental studies of adsorption of amino acids on copper surfaces}

It has been reported that the adsorption of L-lysine on $\mathrm{Cu}(100)$ surface can cause step bunching to form homochiral $\mathrm{R}-\left\{\begin{array}{lll}3 & 1 & 17\end{array}\right\}$ facets following annealing at $430 \mathrm{~K}$ for 20 minutes [3]. Gellman's group has explored the possibility of using chemical means to imprint chirality into metal surfaces and this represents such a system. To initiate the study of enantioselective adsorption on such surfaces, the surface chemistry of L-lysine has been studied on the $\mathrm{Cu}(100)$ surface. In addition the work in Gellman's laboratory has studied the surface chemistry of L-alanine on the $\mathrm{Cu}(110)$ surface as this is another amino acid that is known to form ordered structures when adsorbed on $\mathrm{Cu}$ surfaces.

Both L-lysine on the $\mathrm{Cu}(100)$ surface and L-alanine on the $\mathrm{Cu}(110)$ surface adsorb in the form of deprotonated carboxylate species. Molecular adsorption occurs at low temperature and molecular desorption occurs during heating. This is observed in the temperature range $300-350 \mathrm{~K}$ in the TPD spectra of Figure 5. The remaining monolayer is believe to be in the form of the deprotonated carboxylate. In the case of Lalanine on the $\mathrm{Cu}(110)$ surface one can observe the formation of an ordered overlayer which generates the LEED pattern illustrated in Figure 6. This corresponds to the $\left[\begin{array}{ll}2 & \overline{2} \\ 5 & 3\end{array}\right]$ overlayer lattice that has been observed and reported previously [4]. During subsequent heating the carboxylate layer decomposes at temperatures above $500 \mathrm{~K}$ with 'explosive' kinetics. In Figure 5 these 'explosive kinetics are manifested by the peak at $~ 500-550$ $\mathrm{K}$ which has a leading edge that shifts to higher temperature with increasing coverage and yields a final peak that is very narrow.

The observation of 'explosive' decomposition kinetics for L-lysine and L-alanine offers the opportunity to observe enantiospecific decomposition kinetics when these compounds are adsorbed on naturally chiral surfaces such as $\mathrm{Cu}(643)^{\mathrm{R} \& \mathrm{~S}}$. This is currently under exploration.

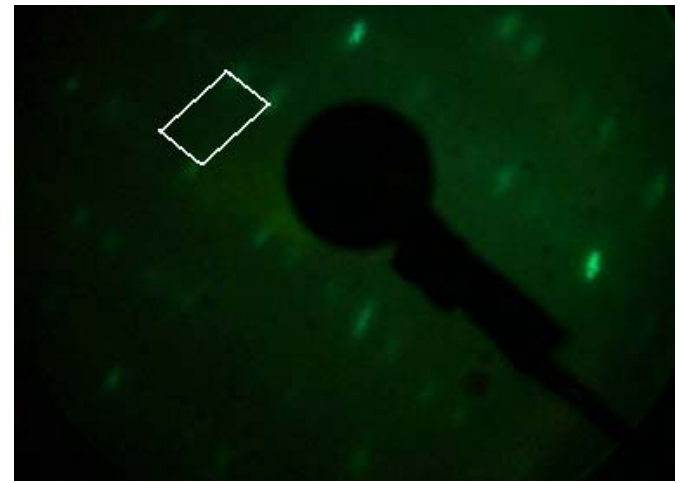

Figure 4. LEED pattern generated by L-alanine on the $\mathrm{Cu}(110)$ surface. This arises from the $\left[\begin{array}{ll}2 & 2 \\ 5 & 3\end{array}\right]$ overlayer formed after adsorption and heating to $400 \mathrm{~K}$.

Figure 5. TPD spectra of L-alanine on the $\mathrm{Cu}(110)$ surface. Molecular desorption occurs in the temperature range $300-350 \mathrm{~K}$. Decomposition occurs with 'explosive' kinetics in the temperature range $500-550 \mathrm{~K}$.

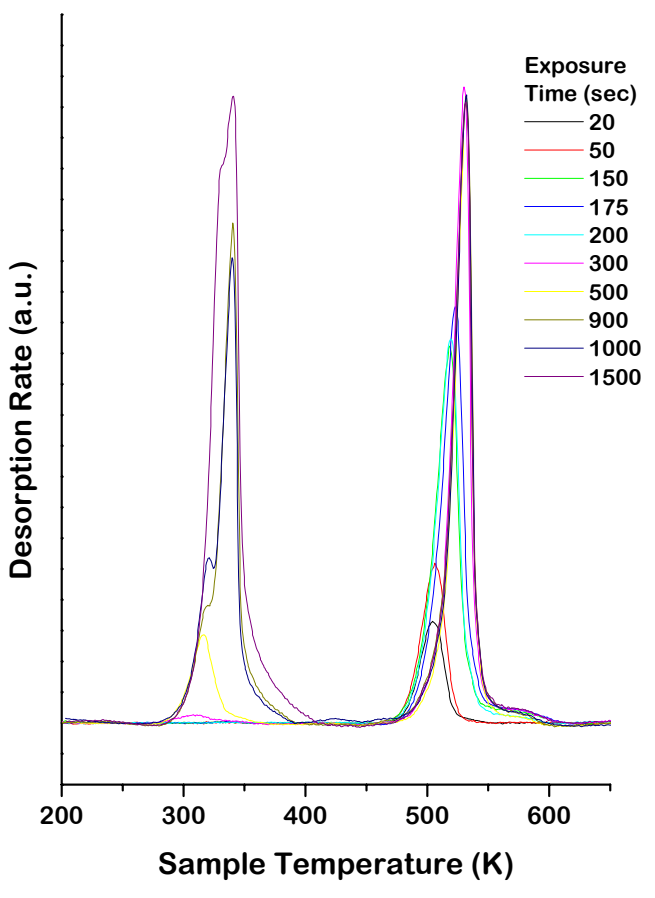




\subsection{Theoretical studies of amino acid adsorption on $\mathrm{Cu}$ surfaces}

Density Functional Theory (DFT) calculations can play a useful complementary role to experimental studies of amino acids on metal surfaces. For example, DFT calculations can be used to distinguish between possible adlayer structures that are challenging to resolve using experiments alone. Sholl's group used calculations of this type to resolve the experimental controversy that existed in terms of the structure of glycine on $\mathrm{Cu}(110)$ [5]. The structure of alanine on $\mathrm{Cu}(110)$ was determined via calculations to be very similar to that of glycine, as might be expected [6]. The structure from these calculations was subsequently found to be in excellent agreement with high resolution experimental data [7].

We performed a systematic series of calculations to predict the structures of glycine, enantiopure alanine, and racemic alanine on both $\mathrm{Cu}(110)$ and $\mathrm{Cu}(100)$ [8]. In each case we considered dense adlayers of dehydrogenated adsorbates, as these are the structures most commonly studied in experiments. Our results are summarized below in Table 2. Even this relatively simple collection of adsorbates and surfaces shows a fascinating range of possible behaviors. Glycine and enantiopure alanine on $\mathrm{Cu}(110)$ both have a single favored structure, although this structure has two orientationally distinct domains on the surface. It is these rotationally distinct domains that were misinterpreted in early STM experiments as providing evidence for the existence of two distinct adlayers structures. If an adlayer of racemic alanine is formed on $\mathrm{Cu}(100)$, the most favored structure is one in which each the distribution of the two enantiomers within the adlayer is random. This situation is referred to as a pseudo-racemate. $\mathrm{On} \mathrm{Cu}(100)$, glycine has two distinct adlayers that are almost equivalent in energy. This situation also occurs for enantiopure alanine. If racemic alanine is used on $\mathrm{Cu}(100)$, however, a single racemate structure is preferred in which one of each enantiomers of the adsorbed molecules appears in each $(2 \times 2)$ surface unit cell. It is tempting to describe the preference for one adlayer structure over another in terms of a single factor such as the number of hydrogen bonds formed within an adlayer. Careful analysis of all of the adlayers we considered on these surfaces, however, indicates that such a simple picture is not valid. Instead, the subtle interplay between hydrogen bonding on the surface, intramolecular distortion in accommodating surface bonding, and the strength of bonds to the surface is the determining factor in dictating which adlayer is ultimately preferred.

Table 2: A summary of the favored adlayers for glycine and alanine on $\mathrm{Cu}(110)$ and $\mathrm{Cu}(100)$ as predicted by DFT. The number of domains is the number of orientationally distinct versions of an individual adlayer available on the surface multiplied by the number of geometrically distinct adlayers coexisting on the surface.

\begin{tabular}{|c|c|c|c|}
\hline Adsorbate & Surface & Favored adlayer & $\begin{array}{c}\text { No. of distinct } \\
\text { domains }\end{array}$ \\
\hline Glycine & $\mathrm{Cu}(110)$ & heterochiral & 2 \\
\hline Glycine & $\mathrm{Cu}(100)$ & $\begin{array}{c}\mathrm{c}(4 \times 2) \text { homochiral } \\
+(2 \times 2) \text { pseudo- } \\
\text { heterochiral }\end{array}$ & 8 \\
\hline Alanine & $\mathrm{Cu}(110)$ & heterochiral & 2 \\
\hline
\end{tabular}




\begin{tabular}{|c|c|c|c|}
\hline (enantiopure) & $\mathrm{Cu}(110)$ & $\begin{array}{c}\text { pseudoracemate- } \\
\text { heterochiral }\end{array}$ & 2 \\
\hline Alanine (racemic) & $\mathrm{Cu}(100)$ & $\begin{array}{c}\mathrm{c}(4 \times 2) \text { homochiral } \\
+(2 \times 2) \text { pseudo- } \\
\text { heterochiral }\end{array}$ & 8 \\
\hline $\begin{array}{c}\text { Alanine } \\
\text { (enantiopure) }\end{array}$ & $\mathrm{Cu}(100)$ & $\begin{array}{c}\text { racemate }(2 \times 2) \\
\text { pseudo-heterochiral }\end{array}$ & 4 \\
\hline Alanine (racemic) & \\
\hline
\end{tabular}

Our primary recent focus in this area has been to use DFT calculations to understand the reconstructions of $\mathrm{Cu}(100)$ that are observed when amino acids are deposited on this surface. As mentioned in section 2.2, a number of experiments have reported the spontaneous appearance of $\mathrm{Cu}\{3,1,17\}$ facets when amino acids are deposited on $\mathrm{Cu}(100)$ surfaces. These observation pose at least two challenges. First, why is it energetically favorable for adsorbed amino acids to reconstruct the flat $\mathrm{Cu}(100)$ surface into a surface with a high step density? Second, why are $\mathrm{Cu}\{3,1,17\}$ facets selected among the very large number of other stepped surface facets that could potentially participate in this process?

We first considered the second question posed above on purely geometric grounds[9]. It is known from experiments and also our earlier calculations that glycine and alanine for dense adlayers on $\mathrm{Cu}(100)$ with two molecules in each $(4 \times 2)$ surface unit cell. Qualitatively, a stepped surface that is a reasonable candidate for forming a favorable surface reconstruction from this adlayer will also have the surface densely covered with adsorbed molecules. If we ask whether a related adlayer can form on a stepped $\mathrm{Cu}(h, k, l)$ surface, a reasonable approach is to seek surfaces with (100)-oriented terraces with unit cell areas and shapes similar to the $(4 \times 2)-\mathrm{Cu}(100)$ unit cell. This criterion does not guarantee that an adlayer on the stepped surface will be energetically favorable relative to the flat surface, but it seems unlikely that stepped surfaces that do not meet this criterion would be viable candidates for an adsorption-driven surface reconstruction.

In considering potential stepped surfaces for adsorption of glycine or alanine, we restricted our interest to intrinsically chiral $\mathrm{Cu}$ surfaces with (100)-oriented terraces. This approach excludes stepped surfaces with atomically straight step edges. The complete set of Miller indices that meets these conditions can be generated using the rules that exist for classifying the chirality of stepped metal surfaces. We have examined a large number of chiral surfaces and for each surface we calculated the surface cell area in the plane perpendicular to the surface normal and two measures of the shape of the unit cell. First, we defined $\alpha_{1}$ to be the ratio of the length of the unit cell vectors with the vectors chosen so $\alpha_{1}<1$. Second, we defined $\alpha_{2}$ to be the ratio of the perimeter of the unit cell to the unit cell area. In each case, the unit cell was defined by constructing the parallelogram connecting four adjacent kink atoms on the surface of interest. Finally, the number of (100) vicinal cells contained in the chiral surface's terrace was calculated. By combining these three factors, a comparison can be established between the $(4 \times 2)$ unit cell of $\mathrm{Cu}(100)$ and the $\mathrm{Cu}(h, k, l)$ surfaces. Table 3 lists the geometric parameters defined above 
for 15 chiral $\mathrm{Cu}(h, k, l)$ surfaces, along with the parameters for $(4 \times 2) \mathrm{Cu}(100)$. Many other chiral surfaces were also examined that showed less similarity with (4x2) $\mathrm{Cu}(100)$; for clarity these are not listed in the table. For the chiral surfaces listed in Table 1 the geometric parameters for $\mathrm{Cu}(h, k, l)^{\mathrm{R}}$ and $\mathrm{Cu}(h, k, l){ }^{\mathrm{S}}$ are identical, so the $\mathrm{R} / \mathrm{S}$ designation in this context is unnecessary.

The results in Table 3 indicate that the unit cell of $\mathrm{Cu}(3,1,17)$ is more similar in size and shape to the $(4 \times 2)$ unit cell of $\mathrm{Cu}(100)$ than any of the other chiral surfaces with similar surface areas. The surface area of the $\mathrm{Cu}(3,1,17)$ unit cell is $8 \%$ larger than that of the flat surface. This makes the $\mathrm{Cu}(3,1,17)$ a more likely outcome from surface reconstruction that chiral surfaces with larger unit cells such as $\mathrm{Cu}(3,1,19)$, which has an area $20.5 \%$ larger than the flat surface. There are two surfaces listed in Table 1 that have unit cell areas closer to the flat surface than $\mathrm{Cu}(3,1,17)$, but in both cases the shape of these unit cells is quite different than the $(4 \times 2)$ unit cell of $\mathrm{Cu}(100)$.

\begin{tabular}{|ccc|ccc|c|}
\hline $\boldsymbol{h}$ & $\boldsymbol{k}$ & $\boldsymbol{I}$ & $\alpha_{1}$ & $\alpha_{2}\left(\AA^{-1}\right)$ & Area $\left(\AA^{2}\right)$ & \# of (100) cells \\
\hline 3 & 1 & 12 & 0.75 & 0.63 & 42.57 & 9 \\
4 & 1 & 11 & 0.45 & 0.98 & 44.44 & 7 \\
3 & 1 & 15 & 0.62 & 0.60 & 50.22 & 6 \\
5 & 1 & 19 & 0.83 & 0.63 & 53.66 & 7 \\
2 & 1 & 8 & 0.84 & 0.55 & 54.37 & 6 \\
$\mathbf{3}$ & $\mathbf{1}$ & $\mathbf{1 7}$ & $\mathbf{0 . 5 8}$ & $\mathbf{0 . 5 5}$ & $\mathbf{5 6 . 6 9}$ & $\mathbf{7}$ \\
5 & 3 & 17 & 0.73 & 0.62 & 58.87 & 6 \\
3 & 1 & 19 & 0.51 & 0.53 & 63.18 & 8 \\
7 & 1 & 19 & 0.53 & 0.59 & 66.38 & 6 \\
7 & 3 & 19 & 0.56 & 0.64 & 66.94 & 6 \\
3 & 1 & 21 & 0.45 & 0.53 & 69.67 & 9 \\
5 & 3 & 21 & 0.64 & 0.49 & 71.46 & 8 \\
7 & 3 & 21 & 0.42 & 0.53 & 73.08 & 7 \\
3 & 1 & 23 & 0.43 & 0.50 & 76.14 & 10 \\
7 & 1 & 23 & 0.53 & 0.50 & 78.79 & 8 \\
\hline 1 & 0 & 0 & 0.50 & 0.59 & 52.43 & 8 \\
\hline
\end{tabular}

Table 3: Geometric data for 15 chiral $\mathrm{Cu}(h k l)$ surfaces with (100)-oriented terraces. See text for definitions. Data for the $(4 \times 2)$ unit cell of the (100) surface is presented at the bottom. Surfaces are sorted by projected surface cell area.

We now turn to assessing the energetic driving force for amino acid induced reconstruction of $\mathrm{Cu}(100)$. To assess this quantity, we must determine the structure of adsorbed amino acids on $\mathrm{Cu}(3,1,17)$ surfaces. This is a challenging task because essentially no information other than the geometric information outlined above can be inferred about these structures from extant experimental data. We first considered the adsorption of glycine (and subsequently alanine) on the step edge of the $\mathrm{Cu}(3,1,17)$ surface [9]. We developed an approach to generating trial structures on this surface that systematically examined the various bonding configurations of the molecules on the 
surface. Figure 6 shows the three lowest energy structures determined in this way for glycine $/ \mathrm{Cu}(3,1,17)$. All three structures exhibit tridentate bonding to the surface, but the specific atoms on the surface to which the adsorbate bonds are different for each structure. Similar calculations were performed for alanine. In this case, the two enantiomers of alanine must be considered separately, because the underlying surface is intrinsically chiral. The energy difference between the preferred structure for the $\mathrm{R}$ enantiomer and the $\mathrm{S}$ enantiomer was found to be only $0.03 \mathrm{eV} /$ molecule. This observation is consistent with experimental observations that, unlike the adsorption of lysine on the same surface, the $\mathrm{Cu}(3,1,17)$ facets that appear following adsorption of alanine on $\mathrm{Cu}(100)$ are not homochiral.
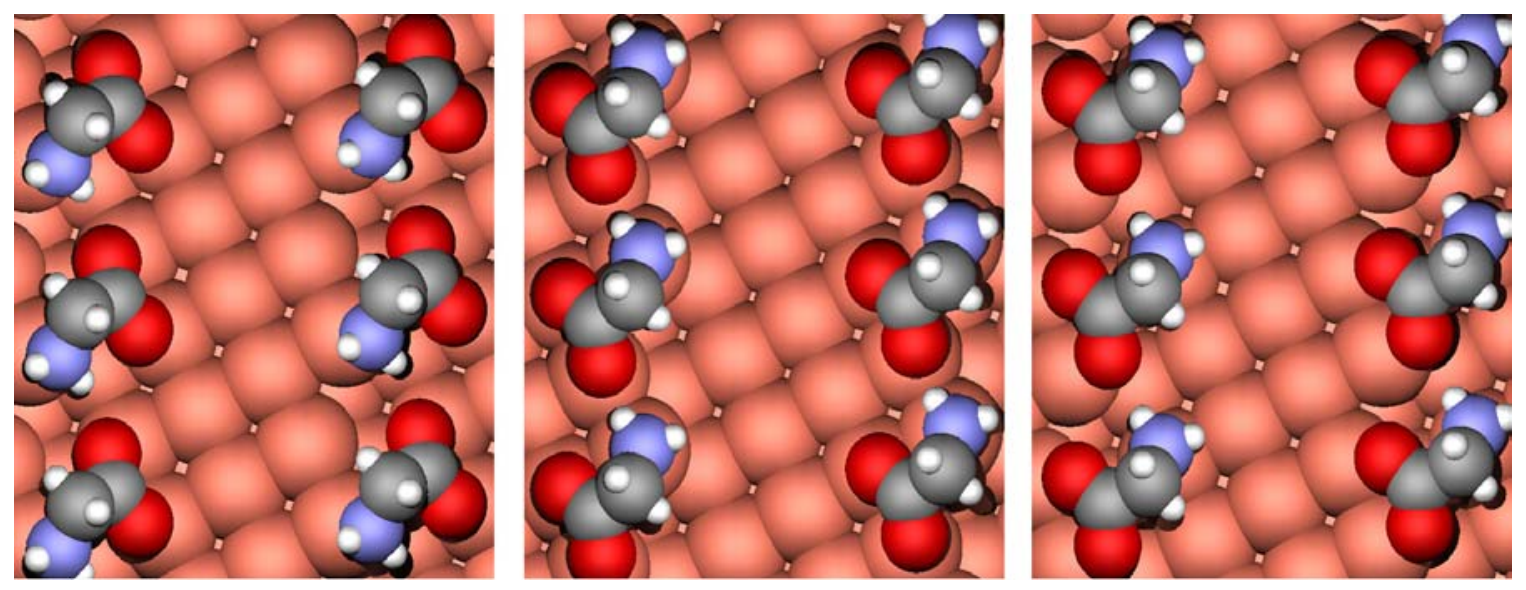

Figure 6: The three most stable bonding configurations of $\mathrm{Gly}$ on $\mathrm{Cu}(3,1,17)^{\mathrm{S}}$, with the lowest energy structure on the left.

The structures shown in Fig. 6 are not sufficient to consider the energetic driving force underlying the reconstruction of $\mathrm{Cu}(100)$ by adsorbed amino acids because this reconstruction involves a dense adlayer on the surface. We performed an extensive series of calculations to generate dense adlayers from low energy structures such as those shown in Fig. 6 for both glycine, enantiopure alanine, and racemic alanine [10]. Figure 7 shows an example of the results of these calculations; specifically, it shows the preferred structure of a dense glycine adlayer on $\mathrm{Cu}(3,1,17)$.

Once the dense adlayers such as the one shown in Fig. 7 were established, the relative energy of the reconstructed surface and the initially flat surface could be considered. To examine the driving force for faceting of $\mathrm{Cu}(100)$ into $\mathrm{Cu}(3,1,17)$ facets due to amino acid adsorption, we note that such a transition increases the surface area of the surface by $1 / \cos (\theta)$, where $\theta$ is the angle between the two surfaces. $\mathrm{For} \mathrm{Cu}(100)$ and $\mathrm{Cu}(3,1,17)$ facets, $1 / \cos (\theta)=1.017$. The driving force for surface faceting can therefore be expressed as

$$
\Delta \sigma=\widetilde{\sigma}_{100}-\frac{\widetilde{\sigma}_{3,1,17}}{\cos (\theta)},
$$

where $\widetilde{\sigma}$ is the surface energy of the surface of interest in the presence of the adsorbed 
adlayer. The relative surface energies calculated using this approach are summarized below in Table 4 . They confirm that in general it is energetically favorable for adsorbed glycine and alanine for reconstruct the $\mathrm{Cu}(100)$ surface into $\mathrm{Cu}(3,1,17)$ facets, despite the energy cost associated with creating the high density of surface steps on the latter surface. The energy cost is compensated by the enhanced binding energy of the adsorbed molecules on the stepped surface, particularly among the molecules that adsorbed directly on the step edge. The fact that reconstructions like this can take place is not in itself surprising, but these calculations provide unprecedented insight into the detailed structures on the surfaces that allow them to happen. As with the range of adlayers we examined on the flat surfaces, the preference for the lowest energy adlayers relative to all the other possible variations that can be considered does not arise from a single contribution (say, hydrogen bonding). Rather, the interplay of all the factors that contribute to the total energy of the adlayer, including hydrogen bonding, surface bonding, and molecular deformations, all play a crucial role in the final energy.

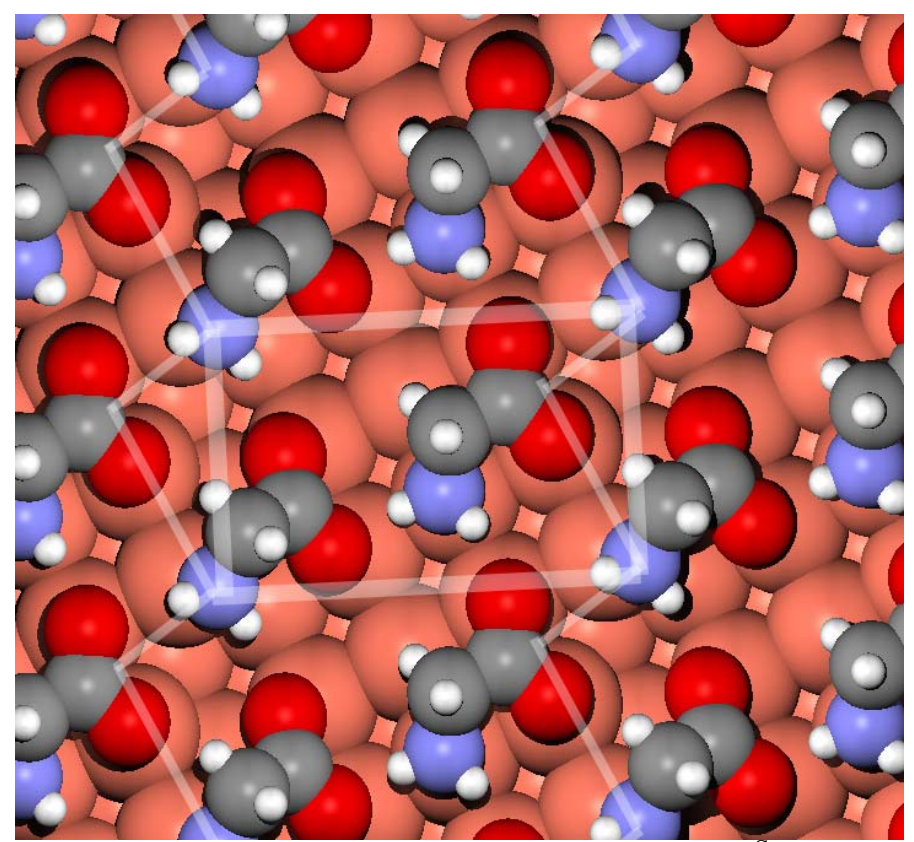

Figure 7: A top view of a dense glycine adlayer on $\mathrm{Cu}(3,1,17)^{\mathrm{S}}$. Thick (thin) white lines denote a surface unit cell (the surface step edge). This configuration is the most energetically stable glycine adlayer identified in our calculations. 


\begin{tabular}{|c|c|c|c|}
\hline $\begin{array}{c}\text { Adlayer on } \\
\text { Cu(3,1,17) }\end{array}$ & Coverage & $\begin{array}{c}\Delta \sigma\left(\mathbf{J} / \mathbf{m}^{2}\right) \\
\text { (lower bound) }\end{array}$ & $\begin{array}{c}\Delta \sigma\left(\mathbf{J} / \mathbf{m}^{2}\right) \\
\text { (upper bound) }\end{array}$ \\
\hline Gly & Moderate & -0.004 & 0.026 \\
\hline Gly & Dense & 0.074 & 0.104 \\
\hline S-Ala & Moderate & -0.006 & 0.023 \\
\hline$R$-Ala & Moderate & -0.017 & 0.013 \\
\hline S, S-Ala & Dense & 0.029 & 0.059 \\
\hline S,R-Ala & Dense & 0.002 & 0.032 \\
\hline
\end{tabular}

Table 4: Relative surface energies of moderate and dense coverage Gly and Ala adlayers on $\mathrm{Cu}(3,1,17)^{\mathrm{S}}$ compared to analogous adlayers on $\mathrm{Cu}(100)$.

\subsection{Theoretical studies of molecular structure in disordered adlayers}

The amino acid adlayers that were described in section 2.4 are strongly ordered. This has obvious advantages in terms of probing the adlayers using electron diffraction and also with DFT calculations. In many of the examples of interest in chiral catalysis, however, the adsorption of chiral molecules onto a surface results only in disordered adlayers. The adsorption of propylene oxide and/or butanoxide on fcc(111) surfaces falls into this class (see section 2.1). Despite the disordered nature of these adlayers, understanding the structure of the adsorbed molecules is of great importance in developing an atomic-scale view of how the chirality of the adsorbed molecules can lead to enantiospecific processes of interest. Motivated by this idea, we have been using DFT calculations in collaboration with experimental studies by Tysoe's group to assign the structure of probe molecules in disordered layers. This approach relies on the methods developed by Tysoe and coworkers to use I-V analysis of the diffuse LEED spots from disordered adlayers to probe the structure experimentally[11]. In practice, applying this approach to a molecule with more than a handful of degrees of freedom requires a starting structure (or structures) that can be refined with the I-V analysis. DFT calculations can play a helpful role in this process by providing these initial structures.

Our initial efforts to coordinate our experimental and theoretical efforts focused on the structure of formate on $\operatorname{Pd}(111)[11]$. Formate is not a chiral molecule, but its functionality is related to those of chiral amino acids, so it is a useful starting point for our approach. More importantly, it is a sufficiently small molecule that the LEED analysis could be performed without using DFT calculations as input. This means that the results from the experimental approach and the DFT calculations can be directly compared in order to judge whether they are consistent. Several geometrical parameters determined from our experimental approach and our DFT calculations are listed in Table 5. It can be seen that the correspondence between the two methods is excellent. We have also reported similar results for acetate/Pd(111) [12]. 


\begin{tabular}{|c|c|c|}
\cline { 2 - 3 } \multicolumn{1}{c|}{} & $\begin{array}{c}\text { Formate/Pd(111) from } \\
\text { LEED }\end{array}$ & Formate/Pd(111) from DFT \\
\hline $\mathrm{d}(\mathrm{O}-\mathrm{Pd}) / \AA$ & $2.16 \pm 0.06$ & 2.13 \\
\hline $\begin{array}{c}\text { Angle of OCO plane to } \\
\text { surface/ }\end{array}$ & $90 \pm 2$ & 90 \\
\hline $\mathrm{OCO}$ angle $/$ & $130 \pm 5$ & 129 \\
\hline $\mathrm{d}(\mathrm{C}-\mathrm{O}) / \AA$ & $1.26 \pm 0.05$ & 1.27 \\
\hline $\mathrm{d}(\mathrm{C}-\mathrm{H}) / \AA$ & - & 1.1 \\
\hline
\end{tabular}

Table 5: Theoretical and experimental geometrical parameters for formate species on $\operatorname{Pd}(111)$.

We are now extending this approach to a more ambitious collection of molecules that include chiral species such as amino acids. Many of the calculations and experiments for this process are complete, but some analysis of the experimental data still remains before the structures of these molecules can be completely assigned.

\section{DOE Interest}

Fine chemical and pharmaceutical production represents a $>\$ 100$ billion per year industry in which surfaces and heterogeneous catalysts could be used to great effect. In the US very little effort has been put into the development of chiral heterogeneous catalysts and there is very little understanding of the origins of enantioselectivity on chiral surfaces. This program represents on of the most significant collective efforts in the US towards addressing these issues.

Enantioselectivity is one of the most subtle forms of chemical selectivity. Catalytic selectivity is an extremely important problem in numerous heterogeneously catalyzed reactions and is of critical importance to a number of DOE technologies. Our efforts to understand and ultimately control enantioselectivity may well lead to a greater or deeper understanding of factors that effects chemical selectivity in general.

\section{Interactions among Groups}

The research in this project has greatly benefited from collaboration between our two research groups and Carnegie Mellon University and with Eddy Tysoe's group at University of Wisconsin Milwaukee and Francisco Zaera's group at University of California Riverside. The studies of chiral templating (section 2.1) were done in close coordination with similar studies by Tysoe and Zaera. The DFT calculations examining the structure of molecules in disordered adlayers on metal surfaces (section 2.4) were performed in direct collaboration with Tysoe. Additionally, initial calculations have been performed in collaboration with Zaera to understand the structure of complex chiral modifiers such as cinchonidine. 


\section{Literature Cited}

1. D. Stacchiola, L. Burkholder and W.T. Tysoe, Enantioselective Chemisorption on a Chirally Modified Surface in Ultrahigh Vacuum: Adsorption of Propylene Oxide on 2-Butoxide-Covered Palladium(111), J. Am. Chem. Soc. 124(2002) 8984.

2. Ilkeun Lee and Francisco Zaera Enantioselectivity of Adsorption Sites Created by Chiral 2-Butanol Adsorbed on Pt(111) Single-Crystal Surfaces, J. Phys. Chem. B 2005, 109, 12920-12926

3. Xueying Zhao, R.G.Z., W. S. Yang, Scanning Tunneling Microscopy Investigation of L-Lysine Adsorbed on $\mathrm{Cu}(001)$. Langmuir, 2000. 16: p. 98129818

4. S.M. Barlow, S.L., D. Le Roux, J. Williams, C. Muryn, S. Haq, R. Raval, Polymorphism in supramolecular chiral structures of R- and S-alanine on $\mathrm{Cu}(110)$. Surface Science, 2005. 590: p. 243-263.

5. Rees B. Rankin and David S. Sholl, Assessment of Heterochiral and Homochiral Glycine Adlayers on $\mathrm{Cu}(110)$ Using Density Functional Theory, Surface Science, 548 (2004) 301-308

6. Rees B. Rankin and David S. Sholl Structure of enantiopure and racemic alanine adlayers on $\mathrm{Cu}(110)$, Surf. Sci. Lett., 574 (2005) L1-L8

7. G. Jones, L. B. Jones, F. Thibault-Starzyk et al., The local adsorption geometry and electronic structure of alanine on $\mathrm{Cu}(110)$, Surf. Sci. 600 (2006) 1924

8. Rees B. Rankin and David S. Sholl Structures of Enantiopure and Racemic Glycine and Alanine Adlayers on $\mathrm{Cu}(110)$ and $\mathrm{Cu}(100)$ Surfaces, J. Phys. Chem. B, 109 (2005) 16764-16773

9. Rees B. Rankin and David S. Sholl First Principles Studies of Chiral Step Reconstructions of $\mathrm{Cu}(100)$ by Adsorbed Glycine and Alanine, J. Chem. Phys, 124 (2006) 074703

10. Rees B. Rankin and David S. Sholl Structures of Dense Glycine and Alanine Adlayers on Chiral Cu(3,1,17) Surfaces, Langmuir, 22 (2006) 8096

11. T. Zheng, D. Stacchiola, J. James, D. S. Sholl, and W. T. Tysoe The Structure of Formate Species on $\operatorname{Pd}(111)$ Calculated by Density Functional Theory and Determined Using Low Energy Electron Diffraction, Surface Science, 574 (2005) $166-174$ 
12. Joanna James, Dilano K Saldin, T. Zheng, W.T. Tysoe, and David S. Sholl Structure and Binding Site of Acetate on Pd(111) Determined Using Density Functional Theory and Low Energy Electron Diffraction, Catalysis Today, 105 (2005) $74-77$ 